\title{
THE NEARBY, YOUNG, ISOLATED, DUSTY STAR HD 166191
}

\author{
Adam Schneider $^{1}$, Inseok Song ${ }^{1}$, Carl Melis ${ }^{2,6}$, B. Zuckerman ${ }^{3}$, Mike Bessell ${ }^{4}$, Tara Hufford ${ }^{1}$, and Sasha Hinkley ${ }^{5,6}$ \\ ${ }^{1}$ Department of Physics and Astronomy, University of Georgia, Athens, GA 30602, USA; \\ aschneid@physast.uga.edu, song@physast.uga.edu, tara@physast.uga.edu \\ ${ }^{2}$ Center for Astrophysics and Space Sciences, University of California, San Diego, CA 92093, USA; cmelis@ucsd.edu \\ ${ }^{3}$ Department of Physics and Astronomy, University of California, Los Angeles, CA 90095, USA; ben@astro.ucla.edu \\ ${ }^{4}$ Research School of Astronomy and Astrophysics, The Australian National University, Weston Creek, ACT 2611, Australia; bessell@mso.anu.edu.au \\ ${ }^{5}$ Department of Astronomy, California Institute of Technology, Pasadena, CA 91125, USA; shinkley@astro.caltech.edu \\ Received 2013 March 5; accepted 2013 September 4; published 2013 October 17
}

\begin{abstract}
We report an in-depth study of the F8-type star HD 166191, identified in an ongoing survey for stars exhibiting infrared emission above their expected photospheres in the Wide-field Infrared Survey Explorer all-sky catalog. The fractional IR luminosity measured from 3.5 to $70 \mu \mathrm{m}$ is exceptionally high $\left(L_{\mathrm{IR}} / L_{\mathrm{bol}} \sim 10 \%\right)$. Near-diffractionlimited imaging observations with the T-ReCS Si filter set on the Gemini South telescope and adaptive optics imaging with the NIRC2 Lp filter on the Keck II telescope confirmed that the excess emission coincides with the star. Si-band images show a strong solid-state emission feature at $\sim 10 \mu \mathrm{m}$. Theoretical evolutionary isochrones and optical spectroscopic observations indicate a stellar age in the range 10-100 Myr. The large dust mass seen in HD 166191's terrestrial planet zone is indicative of a recent collision between planetary embryos or massive ongoing collisional grinding associated with planet building.
\end{abstract}

Key words: circumstellar matter - stars: individual (HD 166191)

Online-only material: color figures

\section{INTRODUCTION}

The vast majority of debris disks discovered to date are cold $\left(T_{\text {dust }} \leqslant 150 \mathrm{~K}\right)$ and exist at distances of tens to hundreds of AU from their host star, many of which were discovered by the Infrared Astronomical Satellite (IRAS; Chen et al. 2005, 2006; Bryden et al. 2006, 2009; Rhee et al. 2007). Instances of warm dust $(T \geqslant 150 \mathrm{~K})$ are much rarer (Song et al. 2005; Currie et al. 2007; Rhee et al. 2008; Smith et al. 2008; Melis et al. 2010; Morales et al. 2011). A rapid rise of dust luminosity at young stellar ages due to warm dust is predicted by simulations, and is ascribed to the beginning stages of oligarchic growth (Kenyon \& Bromley 2008). Currie et al. (2008a, 2008b, 2009) find a dust luminosity peak around 10-20 Myr for intermediate mass stars. Melis et al. (2010) find the epoch of final mass accretion of terrestrial planets to be 30-100 Myr for solar type stars, and 10-30 Myr for intermediate mass stars (1.5-8.0 $M_{\odot}$ ). Many of these warm dust systems have been found to have multiple components in the radial direction (Morales et al. 2011 and references therein), but when the warm dust is the dominant component in the debris disk, then oftentimes there is no evidence for cooler dust (Melis et al. 2010).

The Wide-field Infrared Survey Explorer (WISE) all-sky survey presents an unprecedented opportunity to explore nearby stars for excess emission at mid-infrared wavelengths indicative of warm dusty circumstellar disks. Because circumstellar disks are ultimately linked to a star's final planetary architecture, their study can provide insight into how planets form and are useful as targets in extrasolar planet searches. We present here an observational study and spectral energy distribution (SED) analysis of the dust surrounding the F8-type star HD166191.

\footnotetext{
6 NSF Fellow.
}

\section{IDENTIFICATION OF HD 166191}

As part of an ongoing effort to identify stars with IR-excess, we have cross-correlated the WISE all-sky survey catalog with the Tycho 2 optical star catalog (Høg et al. 2000). In an attempt to limit the search to nearby stars, we adopted a to-

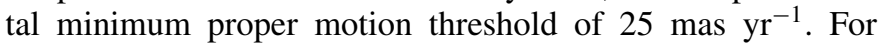
each Tycho star, we fit a synthetic stellar spectrum to the visual (Tycho-2) and near-IR (2MASS) photometric data using a $\chi^{2}$ minimization algorithm following the methods outlined in Rhee et al. (2007). Output from this SED-fitting algorithm yields a significance of excess parameter for each filter band, defined as $\left(F_{\text {meas }}-F_{\text {phot }}\right) / \sigma_{F_{\text {meas }}}$, where $F_{\text {meas }}$ is the measured flux, $F_{\text {phot }}$ is the estimated photospheric flux for the primary stellar type, and $\sigma_{F_{\text {meas }}}$ is the associated measurement uncertainty. Our significance of excess evaluation only takes into account the WISE photometric uncertainties and not any uncertainty from the spectral fitting. Our first inspection, aimed at identifying the largest excess sources, specified a significance of excess greater than 50 in both WISE channel 3 and 4 ( $W 3$ and $W 4$, respectively). This search returned 44 total stars (out of a total sample of $\sim 500,000$ ), the majority of which fell into 1 of 3 categories: pre-main sequence stars (17 total; e.g., HD 282624, HD 142560, and HD 36910), Be stars (10 total; e.g., HD 22191, HR 3858, and HD 105435), and well-studied debris disk hosting stars ( 7 total; e.g., $\beta$ Pic, TW Hya, and BD+20 307). There are 10 potentially new excess sources that need additional confirmation and characterization. Within this sample, though, is HD 166191 (aka HIP 89046, TYC 6843-1557-1), for which there is relatively little information regarding its circumstellar dust. Further inspection of HD 166191 revealed excess at 3.6 and $4.5 \mu \mathrm{m}$ as well. This star was notable amongst other IR-excess candidates because of its large apparent excess and relative obscurity in the literature; this star has largely been ignored by previous searches for circumstellar disks. Due to the little attention paid to this star up to now, we initiated a more 
Table 1

HD 166191 Photometry $^{\mathrm{a}}$

\begin{tabular}{|c|c|c|c|}
\hline Parameter & $\begin{array}{l}\text { Wavelength } \\
\qquad(\mu \mathrm{m})\end{array}$ & $\begin{array}{l}\text { Flux } \\
\text { (Jy) }\end{array}$ & Ref. \\
\hline$B$ & 0.44 & $1.122 \pm 0.021$ & 1 \\
\hline$V$ & 0.55 & $1.646 \pm 0.023$ & 1 \\
\hline$J$ & 1.25 & $1.939 \pm 0.038$ & 2 \\
\hline$H$ & 1.65 & $1.527 \pm 0.060$ & 2 \\
\hline$K_{S}$ & 2.20 & $1.100 \pm 0.018$ & 2 \\
\hline$W 1$ & 3.35 & $0.780 \pm 0.026$ & 3 \\
\hline$W 2$ & 4.60 & $0.874 \pm 0.020$ & 3 \\
\hline$W 3$ & 11.56 & $1.978 \pm 0.026$ & 3 \\
\hline$W 4$ & 22.09 & $3.241 \pm 0.045$ & 3 \\
\hline IRAS & 12.0 & $2.35 \pm 0.75$ & 4 \\
\hline IRAS & 25.0 & $3.80 \pm 0.57$ & 4 \\
\hline$M S X A$ & 8.28 & $2.028 \pm 0.083$ & 5 \\
\hline$M S X C$ & 12.13 & $1.863 \pm 0.117$ & 5 \\
\hline$M S X D$ & 14.65 & $1.953 \pm 0.129$ & 5 \\
\hline$M S X E$ & 21.34 & $3.166 \pm 0.212$ & 5 \\
\hline$A K A R I$ & 18 & $2.466 \pm 0.025$ & 6 \\
\hline IRAC & 3.55 & $0.738 \pm 0.061$ & 7 \\
\hline IRAC & 4.49 & $0.674 \pm 0.033$ & 7 \\
\hline IRAC & 5.73 & $0.872 \pm 0.025$ & 7 \\
\hline IRAC & 7.87 & $1.621 \pm 0.042$ & 7 \\
\hline MIPS & 24 & $2.74 \pm 0.01$ & 8 \\
\hline MIPS & 70 & $1.73 \pm 0.03$ & 8 \\
\hline T-ReCS Si- $1^{\mathrm{b}}$ & 7.73 & $1.51 \pm 0.15$ & 8 \\
\hline T-ReCS Si- $2^{b}$ & 8.74 & $2.44 \pm 0.24$ & 8 \\
\hline T-ReCS Si-3 ${ }^{b}$ & 9.69 & $2.89 \pm 0.29$ & 8 \\
\hline T-ReCS Si-4 ${ }^{b}$ & 10.38 & $3.22 \pm 0.32$ & 8 \\
\hline T-ReCS Si-5 & 11.66 & $2.71 \pm 0.27$ & 8 \\
\hline T-ReCS Si-6 ${ }^{b}$ & 12.33 & $2.00 \pm 0.20$ & 8 \\
\hline
\end{tabular}

Notes.

a Not color-corrected.

${ }^{b}$ Bandwidths for T-ReCS silicate filters 1 through 6 are 0.7, 0.9, 1.0, 1.0, 1.0, and $1.2 \mu \mathrm{m}$, respectively.

References. (1) Johnson magnitudes converted from Tycho 2 (Høg et al. 2000); (2) 2MASS (Cutri et al. 2003); (3) WISE (Cutri et al. 2012); (4) IRAS (Beichman et al. 1988); (5) MSX (Price et al. 2001); (6) AKARI (Ishihara et al. 2010); (7) GLIMPSE (Benjamin et al. 2003); (8) This work.

in depth study of the origin and nature of HD 166191 and it's circumstellar dust.

\section{OBSERVATIONS}

\subsection{Previous Photometry}

After identifying HD 166191 as a WISE excess source, we queried additional catalogs and archives for supplementary photometric measurements. This star was detected at 12, 25, and $60 \mu \mathrm{m}$, with an upper limit at $100 \mu \mathrm{m}$ in the IRAS Point Source Catalog (PSC; Beichman et al. 1988). Inspection of the $60 \mu \mathrm{m}$ detection utilizing the IRAS Scan Processing and Integration (Scanpi) software indicates substantial contamination by nearby IR cirrus, which likely dominates the $100 \mu \mathrm{m}$ upper limit as well. The IRAS Faint Source Catalog avoided the galactic plane and does not contain HD $166191(b=-2$ deg). HD 166191 was also detected by the Midcourse Space Experiment (MSX; Price et al. 2001) at 8.3, 12.1, 14.7, and $21.3 \mu \mathrm{m}$ and the AKARI satellite (Ishihara et al. 2010) at $18 \mu \mathrm{m}$. According to Fujiwara et al. (2013), HD 166191 was observed at $9 \mu \mathrm{m}$ during a South Atlantic Anomaly passage, and hence rejected from the AKARI PSC. 12 and $25 \mu \mathrm{m}$ flux densities from IRAS and at five wavelengths from $M S X$ and $A K A R I$ are provided in Table 1.

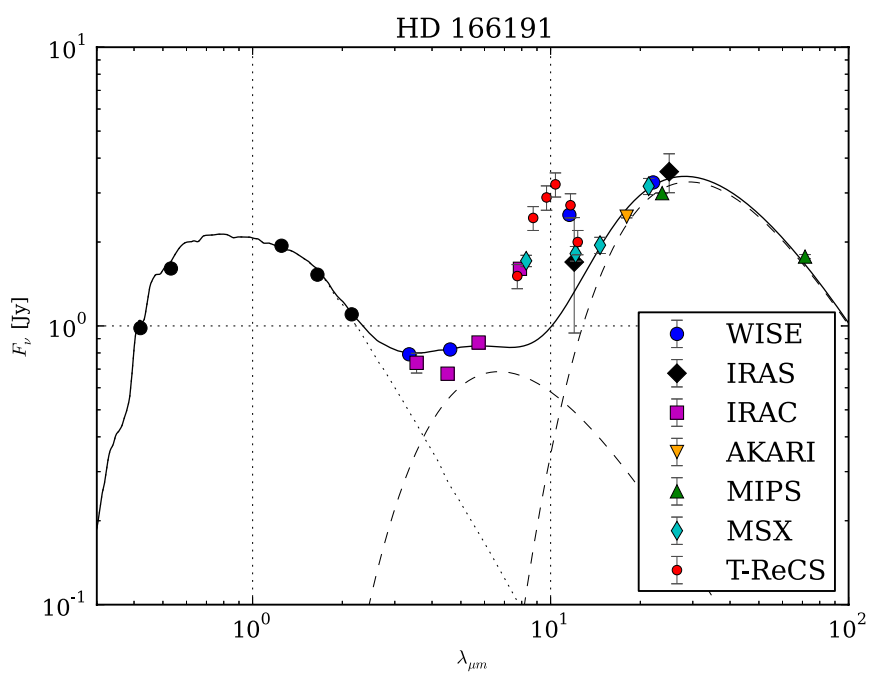

Figure 1. Spectral energy distribution of HD 166191. Black circles represent $B, V, J, H$, and $K$ photometry from Tycho-2 and 2MASS, ordered from left to right. All other symbols are specified in the figure legend. In some cases (IRAC, WISE), the error bars are smaller than the symbol size. The dotted line is the model fit to the Tycho-2 and 2MASS data. The dashed curves are blackbody fits to the excess emission with temperatures of 760 and $175 \mathrm{~K}$. The solid line is the combined stellar and blackbody dust fit. Color corrections have been applied to all broadband photometric measurements greater than $3 \mu \mathrm{m}$.

(A color version of this figure is available in the online journal.)

We also queried the Spitzer Heritage Archive to examine if HD 166191 was observed by any of Spitzer's instruments. The star was observed with the Infrared Array Camera (IRAC; Fazio et al. 2004) as part of the Galactic Legacy Infrared Mid-Plane Survey Extraordinaire (GLIMPSE; Benjamin et al. $2003)$ at $3.6,4.5,5.8$, and $8.0 \mu \mathrm{m}$. HD 166191 was also observed with the Multiband Imaging Photometer for Spitzer (MIPS; Rieke et al. 2004) at 24, and $70 \mu \mathrm{m}$ as part of the MIPSGAL survey (Carey et al. 2009), a MIPS complimentary survey to GLIMPSE. We constructed a mosaic image using the MOsaicker and Point source EXtractor (MOPEX) software (Makarov \& Marleau 2005) at each MIPS wavelength. A standard aperture photometry procedure (aperture radius of 5 pixels and sky annulus of 10-20 pixels) was utilized to extract source counts at each wavelength. IRAC flux densities from the GLIMPSE catalog and calculated MIPS flux densities are listed in Table 1. All fluxes listed in Table 1 are not color-corrected, though they are color-corrected for the creation of Figure 1 (see Section 4.1).

\subsection{Infrared Photometry and Optical Spectroscopy}

We observed HD 166191 on 2012 October 3 UT with an entire set of narrow Silicate filters with T-ReCS (Telesco et al. 1998) at Gemini South (Gemini observation GS-2012B-Q-90-284, Si filter names and central wavelengths given in Table 1). We used the superior mid-IR angular resolution of Gemini South $\left(\sim 0^{\prime \prime} 4\right)$ compared with existing images $\left(>2^{\prime \prime}\right)$, to rule out the presence of an unresolved source of mid-IR contamination (as was found for the reddest Aumann catalogue IRAS source by Lisse et al. 2002), and also to inspect for possible solid state features. The T-ReCS images of HD 166191 were approximately seeing limited and show no evidence of additional sources at any wavelength in the $\sim 20^{\prime \prime} \times 30^{\prime \prime}$ field of view. For the single detected source at the expected position of HD 166191, we measure a FWHM of $\sim 0$ '. 5 in the Si-1 filter image. The measured FWHM 
Table 2

HD 166191 Properties

\begin{tabular}{|c|c|c|}
\hline Parameter & Value & Ref. \\
\hline$\alpha(\mathrm{J} 2000)$ & $18: 10: 30.33$ & 1 \\
\hline$\delta(\mathrm{J} 2000)$ & $-23: 34: 00.3$ & 1 \\
\hline$l\left({ }^{\circ}\right)$ & 7.4400 & 1 \\
\hline$b\left({ }^{\circ}\right)$ & -2.1427 & 1 \\
\hline$\mu_{\alpha}$ & $-3.7 \pm 1.6$ mas yr $^{-1}$ & 2 \\
\hline$\mu_{\delta}$ & $-40.2 \pm 1.5$ mas yr $^{-1}$ & 2 \\
\hline Parallax & $8.39 \pm 1.16 \mathrm{mas}$ & 3 \\
\hline Spectral Type ${ }^{a}$ & $\mathrm{~F} 8 \pm 1$ & 4 \\
\hline$E(B-V)^{\mathrm{b}}$ & 0.09 & 4 \\
\hline$T_{\text {eff }} \mathrm{b}$ & $\sim 6300 \mathrm{~K}$ & 4 \\
\hline $\log g^{b}$ & 3.9 & 4 \\
\hline$[\mathrm{M} / \mathrm{H}]^{\mathrm{b}}$ & -0.25 & 4 \\
\hline RV & $-8.1 \pm 1.3 \mathrm{~km} \mathrm{~s}^{-1}$ & 4 \\
\hline$U V W^{\mathrm{c}}$ & $(-5.6 \pm 1.3,-21.9 \pm 3.0,-8.7 \pm 1.5)\left(\mathrm{km} \mathrm{s}^{-1}\right)$ & 4 \\
\hline Li EW & $120 \pm 5 \mathrm{m \AA}$ & 4 \\
\hline $\mathrm{H} \alpha \mathrm{EW}$ & $2.7 \pm 1 \AA$ & 4 \\
\hline$L_{\mathrm{IR}} / L_{\mathrm{bol}}$ & 0.10 & 4 \\
\hline
\end{tabular}

Notes.

a Spectral type is determined by measuring numerous line depth ratios and utilizing the analytical expressions from Kovtyukh et al. (2003).

${ }^{\mathrm{b}} E(B-V), T_{\text {eff }}, \log g$, and $[\mathrm{M} / \mathrm{H}]$ are determined by fitting model atmosphere fluxes to the WiFeS spectrum.

c $U V W$ space motions are calculated using the measured radial velocity. $U V W$ are defined with respect to the Sun. $U$ is positive toward the Galactic center, $V$ is positive in the direction of Galactic rotation, and $W$ is positive toward the north Galactic pole.

References. (1) 2MASS catalog (Cutri et al. 2003); (2) Tycho 2 catalog (Høg et al. 2000); (3) Hipparcos catalog (van Leeuwen 2007); (4) This work.

is fully consistent with a point source under such observing conditions.

With the Keck II telescope, we obtained adaptive optics images of HD 166191 with the NIRC2 camera $J, H, K_{S}$ filters on 2013 May 1 UT and the $3.8 \mu \mathrm{m}$ (Lp) filter on 2013 May 31 UT. All images were taken with the narrow-field camera (pixel scale $=10 \mathrm{mas}_{\text {pixel }^{-1}}$ ). The noisy lower left quadrant was avoided by employing a three-position dither pattern. Each $J, H$, and $K_{S}$ frame had an exposure time of $1.81 \mathrm{~s}(0.181 \mathrm{~s}$ single frame integration times $\times 10$ coadds), and 6 separate exposures, resulting in total integration times of $10.86 \mathrm{~s}$. The Lp frames had exposure times of $5.3 \mathrm{~s}(0.053 \mathrm{~s}$ single frame integration times $\times 100$ coadds) and 24 separate exposures, resulting in a total integration time of $127.2 \mathrm{~s}$. The data were reduced using custom scripts to subtract the dark current, apply a flat-field correction, shift, and median combine to create final images. The aim of these observations was to clearly show that HD 166191 is unresolved into multiple components and further explore the possible presence of contaminating objects. The results of these observations are discussed in Section 4.2.

To obtain age diagnostics from an optical spectrum, we observed HD 166191 with the Wide Field Spectrograph (WiFeS) and echelle spectrograph at the Siding Spring Observatory's $2.3 \mathrm{~m}$ telescope on 2012 November 03 (UT) and 2012 November 28 (UT), respectively. The measured Li $\lambda 6708$ equivalent width (which is consistent in the WiFeS and echelle spectra), as well as the echelle measured radial velocity, are given in Table 2 and discussed in Section 4.2.

\section{RESULTS}

\subsection{Spectral Energy Distribution}

The SED of HD 166191 as derived from the Table 1 photometry is shown in Figure 1. Every measured flux beyond $3 \mu \mathrm{m}$, which includes measurements from WISE, IRAS, IRAC, MIPS, MSX, AKARI, and T-ReCS (23 measurements in total) lies well above the level expected from a pure stellar photosphere, indicating substantial amounts of circumstellar dust. The shape of the excess emission is complex, and is not easily modeled by a simple blackbody fit, contrary to most known IR-excess sources. While not necessarily a complete or true representation of the system, a simple disk model can aid in estimating dust properties. The excess emission is fit using two blackbodies with temperatures of 760 and $175 \mathrm{~K}$. The solid state dust emission feature around $\sim 10 \mu \mathrm{m}$ influences the photometry from at least four different instruments, including WISE, IRAC, MSX, and T-ReCS and indicates copious amounts of sub-micron- to micron-sized warm dust grains. Color corrections are applied to all broadband photometric measurements at wavelengths greater than $3 \mu \mathrm{m}$ by determining the power law slope at each filter band using the combined stellar and blackbody dust fit and applying the relations found in Wright et al. (2010), the IRC Data User's Manual, ${ }^{7}$ Beichman et al. (1988), the IRAC Instrument Handbook, ${ }^{8}$ the MIPS Instrument Handbook, ${ }^{9}$ and the Midcourse Space Experiment Point Source Catalog Version 1.2 Explanatory Guide. ${ }^{10}$

We note here the discrepancy between the measured Spitzer IRAC flux and WISE flux at $\sim 4.5 \mu \mathrm{m}$. We speculate that this difference is likely due to the higher resolution of the IRAC instrument compared to that of WISE. There is also the possibility that this difference is evidence of temporal variability in the flux at this wavelength, not unlike the variability seen in Meng et al. (2012) and the extreme case of TYC 8241-2652-1 (Melis et al. 2012).

\subsection{The Age of HD 166191}

The age of HD 166191 can give us clues as to whether the dusty disk is primitive or a true debris disk. The age of HD 166191 was estimated following methods in Zuckerman \& Song (2004). We first inspect the position of HD 166191 on a color-magnitude diagram (CMD, Figure 2) and find that it lies above the zero-age main-sequence, consistent with a $3 \sigma$ age range between $\sim 2$ and $15 \mathrm{Myr}$ via comparison with theoretical isochrones from Bressan et al. (2012). Because the presence of an unresolved companion can significantly influence CMD position, leading to an inaccurate age estimate, we investigated for any possible hint of binarity. No indication of double lined binarity is present in the single epoch echelle spectrum of HD 166191. We also sought out any potential companions in available images. The highest resolution publicly available image for HD 166191 in the optical or near-IR comes from the UKIRT Infrared Deep Sky Survey (UKIDSS; Lawrence et al. 2007) Galactic Plane Survey (GPS; Lucas et al. 2008). This image does reveal the presence of a possible companion $\sim 3^{\prime \prime}$ away that is unresolved in 2MASS, though with a $\Delta \mathrm{K}$ of $\sim 4.3$, we do not believe this source is bright enough to significantly

\footnotetext{
7 http://www.sciops.esa.int/SA/ASTROF/docs/IRC_IDUM_1.3.pdf

8 http://irsa.ipac.caltech.edu/data/SPITZER/docs/irac/ iracinstrumenthandbook/

9 http://irsa.ipac.caltech.edu/data/SPITZER/docs/mips/ mipsinstrumenthandbook/

$10 \mathrm{http}: / /$ irsa.ipac.caltech.edu/data/MSX/docs/MSX_psc_es.pdf
} 


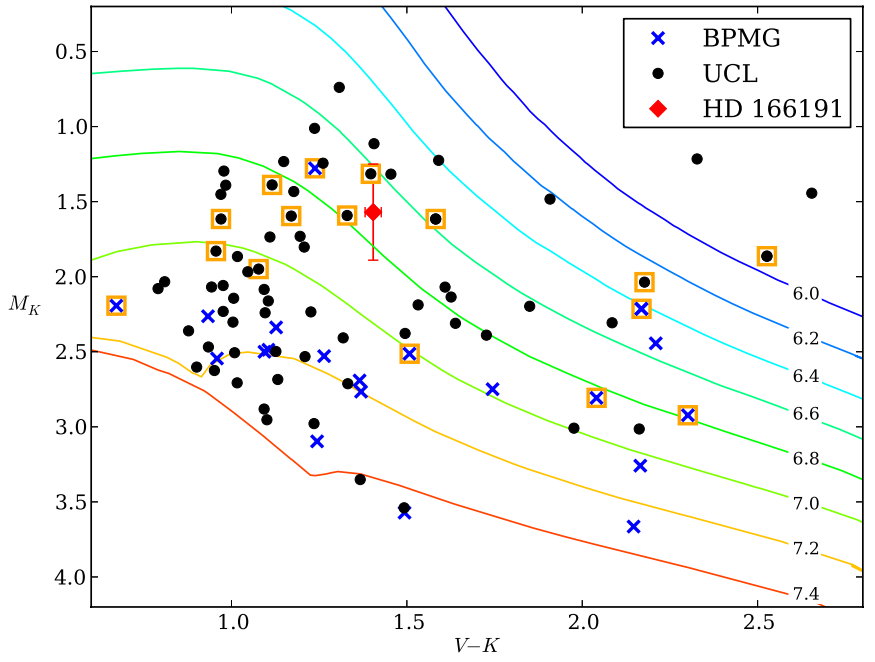

Figure 2. Comparison of the position of HD 166191 on the color-magnitude diagram with theoretical isochrones (Bressan et al. 2012). Isochrones are labeled with $\log (\mathrm{t} / \mathrm{yr})$ values. The black circles are accepted Upper-Centaurus-Lupus (UCL) Hipparcos members from Chen et al. (2011) while the blue crosses are members of the Beta Pictoris Moving Group. Orange boxes indicate known binaries. The error bar for HD 166191 represents the combined photometric and parallax uncertainties.

(A color version of this figure is available in the online journal.)

affect HD 166191's photometry. This source is not present in any of the T-ReCS images because they were not sensitive enough to detect a non-dusty faint star. Keck NIRC2 Lp images reveal the presence of two nearby objects, both approximately $1^{\prime \prime}$ away (Figure 3). The measured flux ratios of these nearby objects to HD 166191 are $\sim 4.1 \%$ and $\sim 0.2 \%$ for " 1 " and " 2 ," respectively.

To evaluate the accuracy of age estimation utilizing theoretical isochrones on a CMD, we compare HD 166191's CMD position with members of the Beta Pictoris Moving Group (BPMG) and the Upper Centaurus Lupus (UCL) region of the Scorpius Centaurus OB association (Figure 2). The BPMG was chosen because it has a well constrained age $(\sim 12 \mathrm{Myr})$ derived from a kinematic traceback analysis (Ortega et al. 2002). The UCL region was chosen because of the possibility that HD166191 is an outlying member (see Section 5.1). Only accepted members of UCL with well-determined parallaxes from Chen et al. (2011) were chosen for the figure. While the non-binary members of the BPMG coincide reasonably well with the $\sim 12 \mathrm{Myr}$ isochrone, the figure indicates that, even for confirmed UCL members with no known binarity, there is significant scatter due to large distance uncertainties. Considering the possibility of being an unresolved multiple system, we very conservatively estimate the CMD age of HD 166191 to be $5_{-3}^{+25}$ Myr.

Lithium content, in addition to being age-dependent, is strongly correlated with stellar mass. The measured equivalent width for HD $166191(120 \pm 5 \mathrm{~mA})$ supports an age $\lesssim 100 \mathrm{Myr}$ (Zuckerman \& Song 2004). We also compare the calculated $U V W$ space motions in Table 2 to those of known nearby stellar associations and find that HD 166191 does lie within the "good box" defined in Zuckerman \& Song (2004) for young stars (see their Figure 6). No X-ray emission from HD 166191 is reported in the ROSAT All-Sky Survey (RASS), but, from the XMMNewton Serendipitous Source Catalog (Watson et al. 2009), we calculate a $\log \left(L_{X} / L_{\text {bol }}\right)$ value of $\approx-4.9$, indicating an age $\gtrsim$ 100 Myr (see Figure 4 of Zuckerman \& Song 2004).

For Sun-like stars, protoplanetary disk dissipation is thought typically to occur by 6 Myr (e.g., Wyatt 2008), although there are

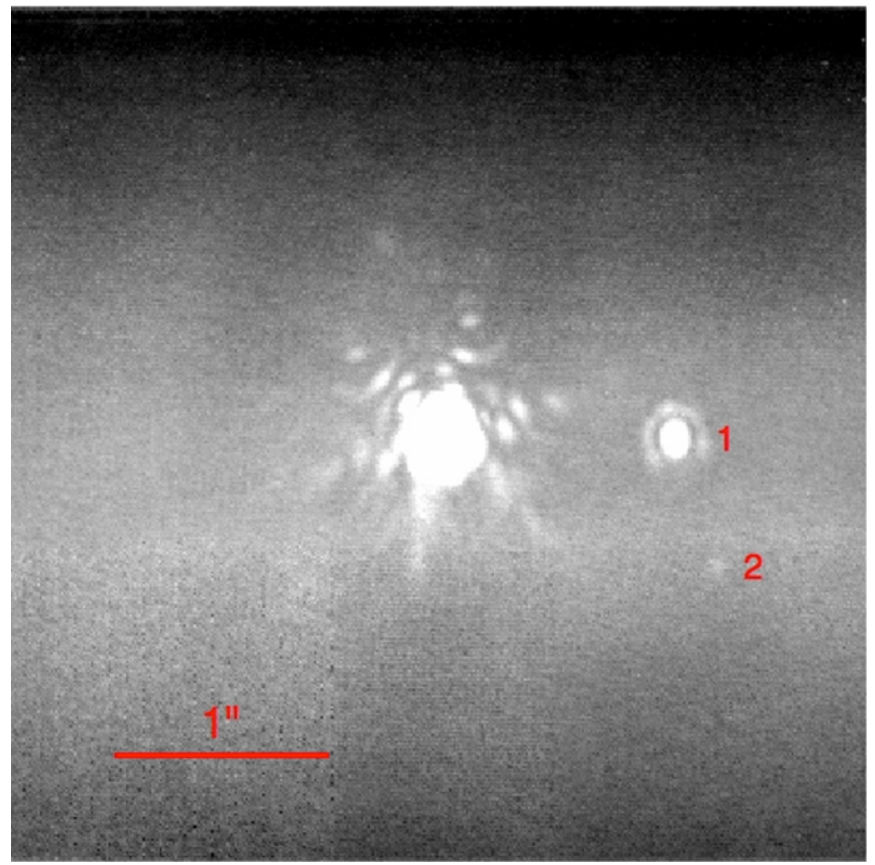

Figure 3. Lp image of HD 166191 obtained with the NIRC2 camera. Additional components (labeled 1 and 2) are discussed in Section 4.2.

(A color version of this figure is available in the online journal.)

exceptions (e.g., TW Hya, HD 98800, V 4046 Sgr). If the disk around HD 166191 is in a primordial or transitional stage, one might expect several observable spectral signatures of active accretion, such as $\mathrm{H} \alpha$ emission; HD 166191 shows no such signs. The $\mathrm{H} \alpha$ absorption equivalent width is given in Table 2. Instead, if HD 1666191 is a weak-line T Tauri star (WTTS) with age $\leqslant 6 \mathrm{Myr}$, we would anticipate strong X-ray emission (X-ray luminosity $>10^{30} \mathrm{erg} \mathrm{s}^{-1}$, or $\log \left(L_{X} / L_{\text {bol }}\right)>-4.3$ for the spectral type of HD 166191; Owen et al. 2011), but this is not the case. Lastly, assuming that the disk is essentially gas free, we evaluate the timescales of small grain survival due to Poynting-Robertson (PR) drag and collisional grinding using Equation (5) from Chen \& Jura (2001) and Equation (5) from Johnson et al. (2012), respectively. Because HD 166191 has a very large fractional infrared luminosity due to the warm dust component ( $\sim 6 \%$; see Section 5.2$)$, the collisional timescale is very short-less than a few years for a range of orbital distances (0.3-1.0 AU). Assuming a grain density of $2.5 \mathrm{~g} \mathrm{~cm}^{-3}$ and a range of grains sizes $(0.1-10 \mu \mathrm{m})$ and orbital distances (0.3-1.0 AU), the range of particle lifetimes due to PR drag is $\sim 100-8000 \mathrm{yr}$. These timescales are much smaller than the age of HD 166191, indicating that, if the disk is gas-free, any primordial dust should have been cleared from it within 10,000 yrs. Thus, any currently present dust must have been created from secondary sources, and then it is not primordial in nature.

Most indicators for HD 166191 are suggestive of a young age. We assign a conservative estimate of 10-100 Myr.

\section{DISCUSSION}

\subsection{Origin of HD 166191}

HD 166191 is too distant $(\mathrm{d} \sim 119 \mathrm{pc}$ ) to belong to any of the nearby young moving groups of comparable age (AB Dor, $\beta$ pic, Tuc-Hor, etc.). However, its distance is similar to that of the Scorpius Centaurus association (ScoCen), and it has a reasonable separation from the historically defined ScoCen 
region to be considered a potential outlying member. It is $\sim 7^{\circ}$ from the nearest edge defining the Upper Centaurus Lupus subregion (based on de Zeeuw et al. 1999). To determine if HD 166191 is a possible UCL outlier, we checked to see if it has $U V W$ space motions consistent with the UCL subgroup. First, we cross-matched the suggested Hipparcos members from de Zeeuw et al. (1999) with the Extended Hipparcos Compilation (XHIP; Anderson \& Francis 2012) to obtain radial velocity measurements, retaining only those members with a radial velocity quality flag of A or B $(\sim 200$ total stars). Using robust statistics (Hoaglin et al. 1983) to exclude possible non-members, we calculated an average $U V W$ for UCL of $(U=-8.4 \pm 2.7, V=-19.1 \pm 3.8$, and $W=-5.5 \pm 2.0)$, values consistent with those found in Chen et al. (2011) $(U=-5.1 \pm 0.6, V=-19.7 \pm 0.4$, and $W=-4.6 \pm 0.3)$. The $U V W$ space motion of HD 166191 $(U=-5.6 \pm 1.3, V=-21.9 \pm 3.0$, and $W=-8.7 \pm 1.5)$ is consistent with UCL space motions, so while this star resides $\sim 7^{\circ}$ from the edge of the UCL boundary defined in de Zeeuw et al. (1999), there is a possible connection.

HD 166191 is located in an extremely crowded field near the galactic center, therefore a companion search based solely on proximity or common proper motion is impractical. For this reason, we searched the area around HD 166191 for any stars showing similar $U V W$ space motions, again utilizing the XHIP catalog. We searched the XHIP catalog within a $5^{\circ}$ radius of $\mathrm{HD} 166191$, requiring Hipparcos distances to be within $20 \mathrm{pc}$ of the distance to HD 166191. We then vetted for any stars within $3 \sigma$ of our measured $U V W$ for HD 166191. Only one star, HD 163296, passed all criteria $(d \sim 119 \mathrm{pc}$, $U=-0.9 \pm 3.3, V=-22.9 \pm 2.2$, and $W=-7.4 \pm 0.8$ ). HD 163296 is a well-studied Herbig Ae star hosting a substantial circumstellar disk with a wealth of silicate features and an estimated age of $5_{-0.6}^{+0.3}$ Myr (Montesinos et al. 2009). This star has previously been proposed to be an outlying ScoCen member (Sitko et al. 2008). While HD 166191 and HD 163296 share similar galactic locations and space motions, HD 163296 show signs of active accretion ( $\mathrm{H} \alpha$ in emission; Silaj et al. 2010) and is detected in X-rays (Günther \& Schmitt 2009), indicating it may be younger than HD 166191. However, it is possible that HD 166191 and HD 163296 are related outlying members of the ScoCen association.

\subsection{Infrared Excess/Silicate Feature}

While HD 166191 is mentioned in Oudmaijer et al. (1992), Fujiwara et al. (2013), and Kennedy \& Wyatt (2013) as an excess star, the full extent of its IR-excess and analysis of the unique shape of its SED has not yet been performed. A planetary or brown dwarf companion could not produce the large IR luminosities seen in Figure 1. We also rule out the possibility that HD 166191 has a transitional disk based on our age estimate in Section 4.2, as transitional disks are dispersed by 10 Myr (Zuckerman et al. 1995; Carpenter et al. 2006; Sicilia-Aguilar et al. 2006; Currie et al. 2009). In addition, the T-ReCs imaging rules out the possibility that the excess infrared emission is produced from interstellar dust grains creating a Pleiades-like effect, as any such emission would have been resolved (e.g., 29 Per; Zuckerman et al. 2012). All available evidence indicates that the excess infrared emission emanates from a single source with a dusty disk. We estimate the fraction of the stellar luminosity reradiated by HD 166191's surrounding dust ( $\tau \equiv L_{\mathrm{IR}} / L_{\mathrm{bol}} \sim 10 \%$ ) by integrating under the stellar SED and dust excess SED curves that appear in Figure 1.
The contribution from the hot component, which includes the $10 \mu \mathrm{m}$ feature, is $\sim 6 \% L_{\text {bol }}$, while the other $\sim 4 \% L_{\text {bol }}$ can be attributed to the cooler, more distant dust component. A detailed mineralogical study will require a mid-IR spectrum. We do note, though, that the peak of the solid-state emission feature $(\sim 10.3 \mu \mathrm{m})$ is indicative of silicates rather than silica, where the latter is typically associated with hypervelocity collisions of large objects (Lisse et al. 2009), implying that the warm dust excess has likely been produced via planetary collisions at velocities less than $5 \mathrm{~km} \mathrm{~s}^{-1}$ or by the gradual grinding of asteroids.

While the two blackbody dust fit model may not be a true interpretation of the dusty nature of this system, we can use this simple model to estimate some fundamental disk parameters. HD 166191 shows evidence of substantial quantities of both warm and cold orbiting dust particles. The substantial amounts of warm dust orbiting HD 166191 are suggestive of ongoing collisional formation of rocky, terrestrial-like planetesimals (Rhee et al. 2008; Melis et al. 2010 and references therein). Assuming the warm dust grains around HD 166191 radiate like blackbodies, they lie at a distance of $\sim 0.3$ AU. Just as with the extremely dusty V488 Per (Zuckerman et al. 2012), this distance from the central star is comparable to that of many transiting planets discovered by NASA's Kepler satellite. Hansen \& Murray (2012) propose a mechanism by which planets form at small distances from their host star without undergoing radial migration. HD 166191 (and V488 Per) can be examples of this type of in situ planet formation. Radiative blowout considerations can constrain the smallest allowable size of orbiting dust particles, which for HD 166191 is $\sim 0.3 \mu \mathrm{m}$. We note that this size assumes no substantial amount of gas and, if the system is as young as $6 \mathrm{Myr}$, this may not then be a good assumption. From Equation (5) of Rhee et al. (2008), the minimum dust mass for the hot dust component $(\tau \sim 6 \%)$ is $5 \times 10^{20}$ to $6 \times 10^{23} \mathrm{~g}$ for the same range of orbital radii and grain sizes mentioned in Section 4.2, equivalent to the mass of a $R=40$ to $400 \mathrm{~km}, 2.5 \mathrm{~g} \mathrm{~cm}^{-3}$ rocky body.

\section{CONCLUSIONS}

We report an in-depth study of the age, origin, and IR-excess of the F8-type star HD 166191. Based on theoretical evolutionary isochrones and optical spectroscopic observations, we estimate an age of 10-100 Myr for this star. Midand far-IR photometry indicates substantial amounts of warm and hot dust, as well as a strong solid-state emission feature at $\sim 10 \mu \mathrm{m}$. The dust encircling HD 166191 is likely due to ongoing planet formation. The fractional infrared luminosity of the HD 166191 system $\left(L_{\mathrm{IR}} / L_{\text {bol }} \sim 10 \%\right)$ is among the largest known, along with V488 Per $\left(L_{\mathrm{IR}} / L_{\text {bol }} \sim 16 \%\right.$; Zuckerman et al. 2012) and TYC 8241-2652-1 before its dust disappeared $\left(L_{\mathrm{IR}} / L_{\text {bol }} \sim 11 \%\right.$; Melis et al. 2012).

Based on observations obtained at the Gemini Observatory, which is operated by the Association of Universities for Research in Astronomy, Inc., under a cooperative agreement with the NSF on behalf of the Gemini partnership: the National Science Foundation (United States), the National Research Council (Canada), CONICYT (Chile), the Australian Research Council (Australia), Ministério da Ciência, Tecnologia e Inovação (Brazil) and Ministerio de Ciencia, Tecnología e Innovación Productiva (Argentina). (Some of) The data presented herein were obtained at the W. M. Keck Observatory, which is operated as a scientific partnership among the California Institute 
of Technology, the University of California and the National Aeronautics and Space Administration. The Observatory was made possible by the generous financial support of the W. M. Keck Foundation. This research has made use of the SIMBAD database and VizieR catalog access tool, operated at CDS, Strasbourg, France. This publication makes use of data products from the Two Micron All Sky Survey, which is a joint project of the University of Massachusetts and the Infrared Processing and Analysis Center/California Institute of Technology, funded by the National Aeronautics and Space Administration and the National Science Foundation, and the Wide-field Infrared Survey Explorer, which is a joint project of the University of California, Los Angeles, and the Jet Propulsion Laboratory/California Institute of Technology, funded by the National Aeronautics and Space Administration. This work is based (in part) on observations made with the Spitzer Space Telescope, which is operated by the Jet Propulsion Laboratory, California Institute of Technology under a contract with NASA. This research made use of data products from the Midcourse Space Experiment. Processing of the data was funded by the Ballistic Missile Defense Organization with additional support from NASA Office of Space Science. This research has made use of the NASA/IPAC Infrared Science Archive, which is operated by the Jet Propulsion Laboratory, California Institute of Technology, under contract with the National Aeronautics and Space Administration. The research was partially supported by NASA grants to UCLA and UGA. C.M. acknowledges support from the National Science Foundation under award No. AST-1003318. A portion of this work was supported by the National Science Foundation under grant No. AST-1203023.

\section{REFERENCES}

Anderson, E., \& Francis, C. 2012, AstL, 38, 331

Beichman, C. A., Neugebauer, G., Habing, H. J., Clegg, P. E., \& Chester, T. J. 1988, Infrared Astronomical Satellite (IRAS) Catalogs and Atlases, Vol. 1, Catalog and Explanatory Supplement

Benjamin, R. A., Churchwell, E., Babler, B. L., et al. 2003, PASP, 115, 953

Bressan, A., Marigo, P., Girardi, L., et al. 2012, MNRAS, 427, 127

Bryden, G., Beichman, C. A., Carpenter, J. M., et al. 2009, ApJ, 705, 1226

Bryden, G., Beichman, C. A., Trilling, D. E., et al. 2006, ApJ, 636, 1098

Carey, S. J., Noriega-Crespo, A., Mizuno, D. R., et al. 2009, PASP, 121, 76

Carpenter, J. M., Mamajek, E. E., Hillenbrand, L. A., \& Meyer, M. R. 2006, ApJ, 651,49

Chen, C. H., \& Jura, M. 2001, ApJL, 560, L171

Chen, C. H., Jura, M., Gordon, K. D., \& Blaylock, M. 2005, ApJ, 623, 493

Chen, C. H., Mamajek, E. E., Bitner, M. A., et al. 2011, ApJ, 738, 122

Chen, C. H., Sargent, B. A., Bohac, C., et al. 2006, ApJS, 166, 351

Currie, T., Kenyon, S. J., Balog, Z., et al. 2008a, ApJ, 672, 558
Currie, T., Kenyon, S. J., Rieke, G., Balog, Z., \& Bromley, B. C. 2007, ApJ, 663, L105

Currie, T., Lada, C. J., Plavchan, P., et al. 2009, ApJ, 698, 1

Currie, T., Plavchan, P., \& Kenyon, S. J. 2008b, ApJ, 688, 597

Cutri, R. M., Skrutskie, M. F., van Dyk, S., et al. 2003, yCat, 2246, 0

Cutri, R. M., et al. 2012, VizieR Online Data Catalog, 2311, 0

de Zeeuw, P. T., Hoogerwerf, R., de Bruijne, J. H. J., Brown, A. G. A., \& Blaaw, A. 1999, AJ, 117, 354

Fazio, G., Hora, J. L., Allen, L. E., et al. 2004, ApJS, 154, 10

Fujiwara, H., Ishihara, D., Onaka, T., et al. 2013, A\&A, 550, 45

Günther, H. M., \& Schmitt, J. H. M. M. 2009, A\&A, 494, 1041

Hansen, B., \& Murray, N. 2012, ApJ, 751, 158

Hoaglin, D. C., Mostellar, F., \& Turkey, F. J. 1983, Understanding Robust and Exploratory Data Analysis (New York: Wiley)

Høg, E., Fabricius, C., Makarov, V. V., et al. 2000, A\&A, 355, L27

Ishihara, D., Onaka, T., Kataza, H., et al. 2010, A\&A, 514, A1

Johnson, B. C., Lisse, C. M., Chen, C. H., et al. 2012, ApJ, 761, 45

Kennedy, G. M., \& Wyatt, M. C. 2013, MNRAS, 433, 2334

Kenyon, S. J., \& Bromley, B. C. 2008, ApJS, 179, 451

Kovtyukh, V. V., Soubiran, C., Belik, S. I., \& Gorlova, N. I. 2003, A\&A, 411,559

Lawrence, A., Warren, S. J., Almaini, O., et al. 2007, MNRAS, 379, 1599

Lisse, C. M., Chen, C. H., Wyatt, M. C., et al. 2009, ApJ, 701, 2019

Lisse, C. M., Schultz, A., Fernandez, Y., et al. 2002, ApJ, 570, 779

Lucas, P. W., Hoare, M. G., Longmore, A., et al. 2008, MNRAS, 391, 136

Makarov, D., \& Marleau, F. 2005, PASP, 117, 1113

Melis, C., Zuckerman, B., Rhee, J. H., \& Song, I. 2010, ApJ, 717, 57

Melis, C., Zuckerman, B., Rhee, J. H., et al. 2012, Natur, 487, 74

Meng, H., Rieke, G., Su, K., et al. 2012, ApJ, 751, L17

Montesinos, B., Eiroa, C., Mora, A., \& Merin, B. 2009, A\&A, 495, 901

Morales, F. Y., Rieke, G. H., Werner, M. W., et al. 2011, ApJ, 730, 29

Ortega, V. G., de la Reza, R., Jilinski, E., \& Bazzanella, B. 2002, ApJ, 575, 75

Oudmaijer, R., van der Veen, W., Waters, L., et al. 1992, A\&AS, 96, 625

Owen, J. E., Ercolano, B., \& Clarke, C. J. 2011, MNRAS, 412, 13

Price, S. D., Egan, M. P., Carey, S. J., Mizuno, D. R., \& Kuchar, T. A. 2001, AJ, 121,2819

Rhee, J. H., Song, I., \& Zuckerman, B. 2008, ApJ, 675, 777

Rhee, J. H., Song, I., Zuckerman, B., \& McElwain, M. 2007, ApJ, 660,1556

Rieke, G. H., Young, E. T., Engelbracht, C. W., et al. 2004, ApJS, 154, 25

Sicilia-Aguilar, A., Hartmann, L., Calvet, N., et al. 2006, ApJ, 638, 897

Silaj, J., Jones, C. E., Tycner, C., Sigut, T., \& Smith, A. D. 2010, ApJS, 187,228

Sitko, M. L., Carpenter, W. J., Kimes, R. L., et al. 2008, ApJ, 678, 1070

Smith, R., Wyatt, M. C., \& Dent, W. R. F. 2008, A\&A, 485, 897

Song, I., Zuckerman, B., Weinberger, A. J., \& Becklin, E. E. 2005, Natur, 436, 363

Telesco, C. M., Pina, R. K., Hanna, K. T., et al. 1998, Proc. SPIE, 3354, 534 van Leeuwen, F. 2007, A\&A, 474, 653

Watson, M. G., Schroder, A. C., Fyfe, D., et al. 2009, A\&A, 493, 339

Wright, E. L., Eisenhardt, P., Mainzer, A., et al. 2010, AJ, 140, 1868

Wyatt, M. C. 2008, ARA\&A, 46, 339

Zuckerman, B., Forveille, T., \& Kastner, J. H. 1995, Natur, 373, 494

Zuckerman, B., Melis, C., Rhee, J. H., Schneider, A., \& Song, I. 2012, ApJ, 752,58

Zuckerman, B., \& Song, I. 2004, ARA\&A, 42, 685 